\title{
Subband occupancies and zero-field spin splitting in InSb-CdTe heterojunctions: magnetotransport experiments and self-consistent calculations
}

\author{
S K Greenet, J Singleton¥\$, P Sobkowicz||, T D Goldingt, \\ M Peppert, J A A J Perenboom\$ and J Dinant \\ † Cavendish Laboratory, University of Cambridge, Madingley Road, \\ Cambridge CB3 OHE, UK \\ ¥ Clarendon Laboratory, Parks Road, Oxford OX1 3PU, UK \\ $\$$ High Field Magnet Laboratory and Research Institute for Materials, University of \\ Nijmegen, NL-6525 ED Nijmegen, The Netherlands \\ || Institute of Physics, Polish Academy of Sciences, 02-668 Warsaw, Al. Lotnikow \\ 32/46, Poland \\ II US Army Center for Night Vision and Electro-Optics, AMSEL-RD-NV-IT, \\ Fort Belvoir, VA 22060-5677, USA
}

Received 23 April 1992, accepted for publication 24 June 1992

\begin{abstract}
A variety of magnetotransport measurements have been performed on InSb-CdTe heterojunctions containing high mobility $\left(\sim 20000 \mathrm{~cm}^{2} \mathrm{~V}^{-1} \mathrm{~s}^{-1}\right)$ two-dimensional electron gases (2DEGS) with low areal carrier densities $\left(N_{s} \sim 1.8\right.$ $4.2 \times 10^{11} \mathrm{~cm}^{-2}$ ). The experimental data have been compared with self-consistent calculations of the energy levels carried out using the 'three-band' model of narrow-gap semiconductors.

The 2OEGS in the samples exhibit well resolved quantum Hall plateaux and Shubnikov-de Haas oscillations at temperatures below $5 \mathrm{~K}$; using these data and the persistent photoconductivity (PPC) effect, the subband occupancies have been evaluated as functions of $N_{\mathrm{s}}$. Parallel-field magnetoresistance measurements show that only two subbands are occupied, even at the highest carrier densities, and indicate the importance of intersubband scattering. The self-consistent calculations predict the number of occupied subbands correctly, and are in good agreement with the experimental subband occupancies when the effects of a small amount of interdiffusion are taken into account.

The low-field magnetoresistance of the heterojunctions is at first positive and then negative; this is characteristic of weak localization in the presence of spin-orbit scattering. An analysis of the data indicates that this is caused by a spin splitting of the subbands at the Fermi energy, present even in zero magnetic field, and of order 2-4 meV. The splitting results from three distinct mechanisms, two of which are included exactly in the self-consistent calculations. The calculations reproduce the size of the observed splitting reasonably well, indicating that these two terms are the dominant contributions to the effect. In addition, the third mechanism, not included in the self-consistent model, has been estimated to give splittings $\sim 1 \mathrm{meV}$. These results illustrate the importance of the zero-field spin splitting in narrow-gap semiconductor space-charge layers.
\end{abstract}

\section{Introduction}

Two-dimensional (2D) systems based on narrow-gap semiconductors (NGS) exhibit many interesting properties absent or significantly weaker in wider-gap systems such as $\mathrm{GaAs}-(\mathrm{Ga}, \mathrm{Al}) \mathrm{As}$ heterostructures, including strong non-parabolicity, interband tunnelling and large spin splitting even in zero magnetic field. The origins of all of these phenomena lie in the strong interaction between the conduction and valence bands [1-4]. 
The 2D narrow-gap systems studied so far have generally been metal-insulator-semiconductor (MIS) structures of InSb, $(\mathrm{Hg}, \mathrm{Cd}) \mathrm{Te},(\mathrm{Hg}, \mathrm{Mn}) \mathrm{Te}$ or $(\mathrm{Hg}, \mathrm{Mn}, \mathrm{Cd}) \mathrm{Te}$, or space-charge layers at surface anodic oxide films or grain boundaries in similar materials (see [5-9] and references therein). Heterojunctions constructed from the narrow-gap III-V semiconductor InSb and the II-VI semiconductor CdTe offer considerable advantages over the above systems from a fundamental physics point of view. The constituent materials have the same crystal structure, and almost the same lattice constants, which should lead to a perfect, almost strain-free interface $[10,11]$. The band structures are also similar, having the same band ordering; the sp momentum matrix elements $P$ are very close in both materials, which suggests that the Bloch basis functions are almost identical [10-12]. This is in stark contrast to the situation in MIS structures or accumulation/inversion layers, where the physical interface may be far from perfect and the band structure of the insulator or overlayer unknown (and possibly very different from the semiconductor). InSb-CdTe heterojunctions therefore offer an ideal testing-ground for the effective mass approximation used in calculations of the energy structure of $2 \mathrm{D}$ systems [1-4], especially in the treatment of the boundary conditions at the interface, which are important in predicting, e.g., the spin splitting of the subbands in zero magnetic field. Furthermore, the interface barrier for the conduction band electrons $(\sim 550 \mathrm{meV})$ is almost twice as high as the InSb band gap, which ensures a good localization of the electrons in the $2 \mathrm{D}$ inversion layer [12]. Thus the InSb-CdTe heterojunction is in many ways a 'better' version of previously investigated NGS systems. (It should be noted that InSb-CdTe heterostructures also possess considerable technological potential; a discussion of this is beyond the scope of this paper, and the reader is referred to [10-12] and references therein.)

Recently, InSb-CdTe heterojunctions exhibiting electron mobilities around $20000 \mathrm{~cm}^{2} \mathrm{~V}^{-1} \mathrm{~s}^{-1}$ have become available for the first time through improvements in growth techniques [12]. In order to address some of the problems mentioned above we have carried out a magnetotransport study of such samples, and performed theoretical self-consistent calculations of the energy levels of InSb-CdTe heterojunctions which have been compared with the experimental data. The Shubnikov-de Haas and quantum Hall effects have been used to measure the subband occupancies of the samples, and the number of occupied subbands and the importance of intersubband scattering have been studied using magnetoresistance experiments with the field applied in the 2D plane. In addition we have employed low-field magnetoconductivity to determine the zero-magnetic-field spin splitting of the subbands, obtaining values $\sim 2-4 \mathrm{meV}$. The self-consistent calculations reproduce the experimental values for the number of occupied subbands and their occupancies well, and the predicted size of the zero-field spin splitting is in satisfying agreement with the measured values.

\section{Experimental details and growth considerations}

The realization of high-quality InSb-CdTe devices has been hindered by growth difficulties, which result in interface defects, cross-doping caused by interdiffusion, and reactions leading to the formation of $\mathrm{In}_{2} \mathrm{Te}_{3}$ at the interface (see [12] and references therein). As a result, the earliest measurements of electrical transport in a $2 \mathrm{DEG}$ in InSb-CdTe heterojunctions revealed low mobilities $\left(\mu<10000 \mathrm{~cm}^{2} \mathrm{~V}^{-1} \mathrm{~s}^{-1}\right)$ and high $2 \mathrm{D}$ carrier densities $N_{\mathrm{s}}$, leading to the occupation of at least three subbands [13]. Subsequent studies reported $\mu \sim 3200 \mathrm{~cm}^{2} \mathrm{~V}^{-1} \mathrm{~s}^{-1}$, with $N_{\mathrm{s}} \sim 4.5 \times 10^{12} \mathrm{~cm}^{-2}$; around six subbands were occupied at those carrier densities [14]. In order to study the underlying physics of the InSb-CdTe system it is desirable to decrease $N_{\mathrm{s}}$, to reduce the number of occupied subbands, and to overcome the growth difficulties leading to poor scattering times and mobilities.

The InSb-CdTe heterojunctions were grown in a Varian $360 \mathrm{MBE}$ system. In order to ensure a large electric field at the interface of the heterojunction, and hence a small number of occupied subbands $[2,5]$, InSb (100) substrates doped p-type to a net acceptor concentration of $N_{\mathrm{A}}=10^{16} \mathrm{~cm}^{-3}$ were used, rather than the lower p-dopings previously employed for InSb MIS structures (e.g. $[2,3,5])$. Prior to loading, the substrates were solvent cleaned, and immediately before growth the native oxide was removed from substrate surfaces by heating at $410^{\circ} \mathrm{C}$ in an $\mathrm{Sb}_{4}$ flux: the latter process avoids the depletion of $\mathrm{Sb}$ and type-conversion probably observed by other authors [14]. Two microns of CdTe was then grown directly onto the substrate at a temperature of $190^{\circ} \mathrm{C}$, using a $3 \times$ excess flux of $\mathrm{Cd}$ over $\mathrm{Te}$, in order to avoid interdiffusion and reaction, and to preserve an abrupt interface; the experimental studies leading to this choice of growth conditions have been described in detail elsewhere [12].

The samples were fabricated into $2 \mathrm{~mm} \times 0.5 \mathrm{~mm}$ Hall bars by defining a pattern using photoresist, and subsequently etching the samples in a solution of $1: 2: 2$ $\mathrm{HNO}_{3}: \mathrm{H}_{2} \mathrm{O}_{2}: \mathrm{H}_{2} \mathrm{O}$ for $5 \mathrm{~s}$. Low-resistance contacts were provided by annealing in small In dots at $180^{\circ} \mathrm{C}$ for 10 minutes. Total 2D carrier densities $N_{\mathrm{s}}$, and mobilities $\mu$, taken from the Shubnikov-de Haas and Hall effect measurements described in section 4 , are shown in table 1 for the five samples studied in this work, which were taken from two wafers. It should be noted that the variation in $N_{\mathrm{s}}$ amongst the samples is due to a gradual increase in carrier density with time with the samples stored at room temperature, rather than inhomogeneities; the measurements described here took place over a period of six months. The values of $N_{\mathrm{s}}$ and $\mu$ are typical, and varied in a non-systematic manner on thermal cycling. Illumination with a red LED at cryogenic temperatures increases $N_{\mathrm{s}}$ : at $1.3 \mathrm{~K}$ this persistent photoconductivity (PPC) remains for several hours, allowing sample parameters to be studied as a function of $N_{\mathrm{s}}$. Table 2 shows the typical effect of illu- 
Table 1. Subband occupancies $\left(N_{0}\right.$ and $\left.N_{1}\right)$, $2 \mathrm{D}$ carrier densities $\left(N_{s}\right)$ and mobilities of the samples used: carrier densities are in units of $10^{11} \mathrm{~cm}^{-2}$, mobilities in $\mathrm{cm}^{2} \mathrm{~V}^{-1} \mathrm{~s}^{-1}$. - denotes saturated values.

\begin{tabular}{lllll}
\hline Sample & $N_{0}$ & $N_{1}$ & $N_{s}$ & Mobility \\
\hline 1 & & & & \\
2 & 1.71 & 0.0 & 1.71 & 16700 \\
3 & 2.06 & 0.27 & 2.33 & 22200 \\
$3^{*}$ & 1.95 & 0.26 & 2.21 & 16000 \\
4 & 2.44 & 0.67 & 3.11 & 17700 \\
$4^{*}$ & 2.39 & 0.26 & 2.65 & 18800 \\
5 & 3.59 & 0.51 & 4.10 & 18300 \\
$5^{*}$ & 2.59 & 0.30 & 2.89 & 16000 \\
& 3.45 & 0.49 & 3.94 & 15700 \\
\hline
\end{tabular}

Table 2. Effect of illumination on $N_{s}$ and mobility for a typical sample; units are as in table 1.

\begin{tabular}{lll}
\hline IIIumination & $N_{\mathrm{s}}$ & Mobility \\
\hline 0 & 2.2 & 18000 \\
$1 \mathrm{~s}$ & 2.4 & 21000 \\
$2 \mathrm{~s}$ & 2.7 & 20000 \\
Saturation & 3.2 & 18000 \\
\hline
\end{tabular}

mination on the sample mobility, which first increases and then decreases.

For most of the transport measurements described below, the samples were mounted on a stage enabling them to be rotated in situ to a precision of $\pm 0.1^{\circ}$; the stage was immersed in ${ }^{4} \mathrm{He}$ exchange gas at a temperature which could be varied between 1.2 and $4.2 \mathrm{~K}$, and the whole cryostat placed in the bore of a $20 \mathrm{~T}$ Bitter magnet. In addition, a simple ${ }^{3} \mathrm{He}$ cryostat for subkelvin temperatures and a continuous flow cryostat for temperatures between 4 and $300 \mathrm{~K}$ were used.

\section{Self-consistent calculations}

The self-consistent calculations are based on the 'threeband' model of a narrow-gap semiconductor and are described briefly in this section: more detailed accounts are given in $[15,16]$. The model treats the interactions between the $\Gamma_{6}, \Gamma_{8}$ and $\Gamma_{7}$ states of the narrow-gap semiconductor exactly, but neglects all the influence of the other states. As a result, the Hamiltonian matrix $(8 \times 8)$ is linear in $k$. Such models have proved useful in the study of earlier 2D systems based on narrow-gap scmiconductors $[1-4,15,16]$, leading to reasonably accurate predictions of the subband occupancies, cyclotron energies and electron $g$-factors. However, because of the uncertainties about the structure of the insulator and interface region in MIS and inversion/accumulation layer systems, it is not possible to give unique theoretical results for effects such as the zero-field spin splitting.

The three-band model leads eventually to a set of two coupled differential equations for the envelope functions of the two spin states corresponding to the $\Gamma_{6}$ conduction band. The potential $V(z)$ is connected to the electron wavefunctions via the Poisson equation.
For narrow-gap semiconductors, where many-body corrections are small, the potential is, as usual, given by the Hartree approximation. The eigenequations and the Poisson equation must be solved self-consistently: the fact that the wavefunctions depend on the wavevector in the plane of the interface means that, in contrast to parabolic (wide-gap) semiconductors, wavefunctions for the whole range of parallel wavevector should be included in the calculation of $V(z)$.

Matching of the envelope functions at the interface is quite natural due to the similarity of the band and crystal structures of InSb-CdTe. A unique set of conditions connecting the envelopes and their derivatives on both sides of the boundary is obtained from the continuity of the total wavefunction and current conservation. These conditions are different for the two spin states, and thus contribute to the zero-field spin splitting. The numerical results of the calculation will be shown in the following sections, along with the relevant data.

\section{High-field magnetotransport}

\subsection{Subband occupancies}

At high temperatures, the conductivity of the samples is dominated by the holes in the p-InSb substrate: however, below $15 \mathrm{~K}$, the Hall coefficient changes sign as the carriers in the bulk InSb and the CdTe epilayer have frozen out, leaving just the electrons in the 2DEG at the heterojunction $[12,17]$. The existence of the $2 \mathrm{DEG}$ is demonstrated in figure 1 , which shows typical magnetotransport data for one of the samples with a low $N_{\mathbf{s}}$ : note that prominent Shubnikov-de Haas $(\mathrm{SdH})$ oscillations are seen in the diagonal resistivity $\rho_{x x}$, and that the $\mathrm{SdH}$ minima become close to zero at higher fields, features characteristic of a single $2 D E G$ with no parallel conduction [18]. The data for the Hall resistivity, $\rho_{x y}$, show broad, accurately quantized Hall effect (QHE) plateaux at these higher fields (see [19] and references therein). At these low values of $N_{\mathrm{s}}$, a comparison of the Hall data and the positions of the SdH oscillations [20], and the field dependence of the Hall coefficient [21] indicate that only one subband is occupied to the limits of experimental accuracy. This assertion is supported by a plot of the extremum index against reciprocal field for the peak and trough positions of the $\mathrm{SdH}$ oscillations (see inset to the upper part of figure 1), which is a straight line extrapolating to the origin.

On moving to samples with higher $N_{\mathrm{s}}$, the $\mathrm{SdH}$ oscillations begin to change in form: the form of the oscillations [20] and comparisons of $\rho_{x x}$ and $\rho_{x y}$ data suggest that a second subband is now populated. Figure 2 shows data for such a sample after illumination with a red LED: the effects of the occupancy of a second subband are plainly visible as a second periodicity in the $\mathrm{SdH}$ oscillations [20].

Subband occupancies $\left(N_{0}, N_{1}\right)$ can be deduced from a careful study of the $\mathrm{SdH}$ minima, $\mathrm{SdH}$ oscillation periodicities and the Hall effect, all performed with the 


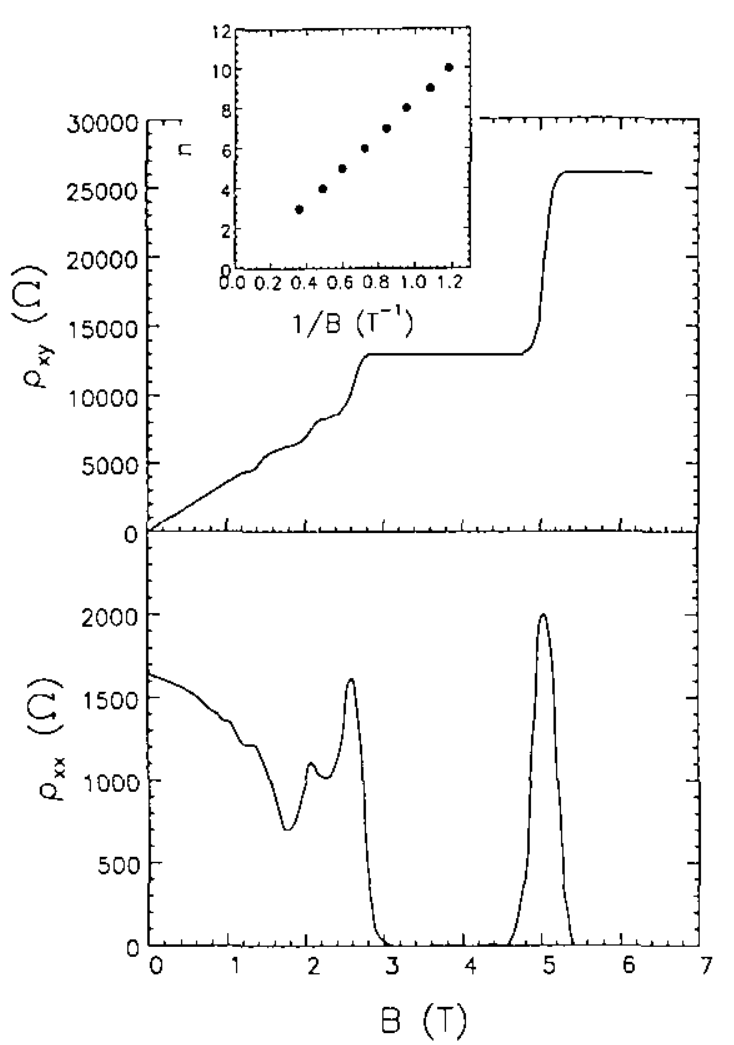

Figure 1. Diagonal magnetoresistivity $\rho_{\alpha \alpha}$ (lower part) and Hall resistivity $\rho_{x y}$ (upper part) for an InSb-CdTe sample with $N_{\mathrm{s}}=1.7 \times 10^{11} \mathrm{~cm}^{-2}$. The inset shows the index of the extrema of the oscillations in $\rho_{x x}$ against reciprocal field.

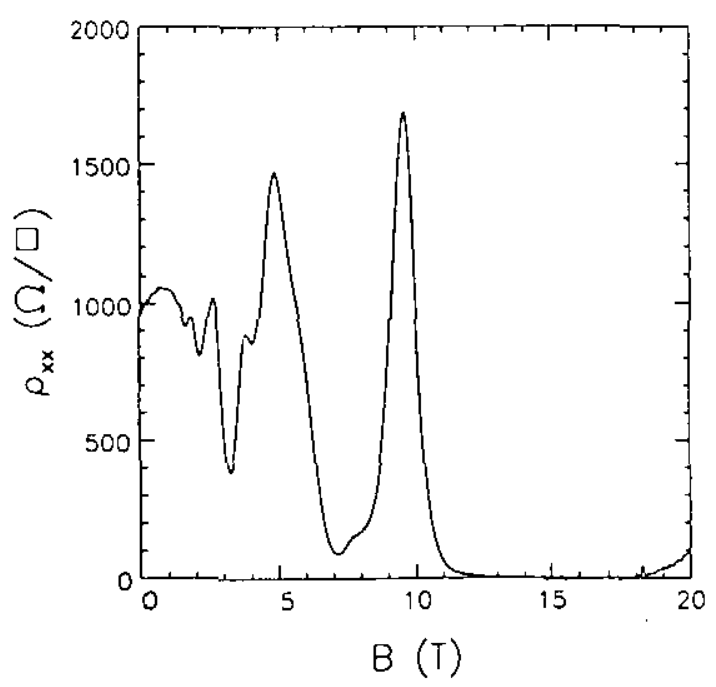

Figure 2. The diagonal magnetoresistivity for an InSb-CdTe sample after saturation with red light $\left(N_{\mathrm{s}}=3.6 \times 10^{11} \mathrm{~cm}^{-2}\right)$.

magnetic field at a range of angles to the sample surface normal $[9,20,22]$. Experimental occupancies are plotted as a function of $N_{\mathrm{s}}$ in figure 3 ; as will be shown in the following section, only two subbands are populated in the samples, even at the highest carrier densities. The different values of $N_{\mathrm{s}}$ were obtained both by using several samples and by using PPC. The data are typical of inversion layers on heavily p-doped narrow-gap semi-

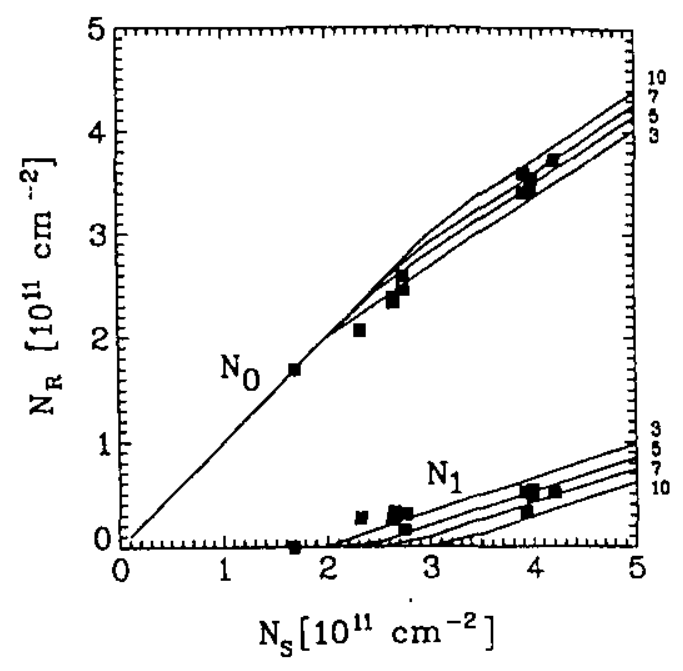

Figure 3. Subband occupancy $\left(N_{0}, N_{1}\right)$ against $\mathrm{N}_{\mathrm{s}}$ for several samples before and after saturation. Experimental values are shown as points, and the results of self-consistent calculations as curves. The error bars for the data (omitted for clarity) are roughly twice the linear dimensions of the square points in both $x$ and $y$ directions. The curves are labelled with the value of $N_{\mathrm{A}}$ (in units of $10^{15} \mathrm{~cm}^{-3}$ ) used in the calculation.

conductors $[2,22]$, in that the population of the $R=1$ subband does not start until a relatively high value of $N_{\mathrm{s}}\left(\approx 1.8 \times 10^{11} \mathrm{~cm}^{-2}\right.$ in this case $)$. In contrast, in narrow-gap accumulation layers, and inversion layers in which the acceptor concentration is low, the occupation of the second subband starts at an almost undetectably small value of $N_{\mathrm{s}}$ [9].

Calculated subband occupancies are shown as lines in figure 3. In general, the occupancies calculated using the nominal value of the bulk InSb acceptor concentration, $N_{\mathrm{A}} \approx 1 \times 10^{16} \mathrm{~cm}^{-3}$, are in passable agreement with the experiment, especially for high values of $N_{\mathrm{s}}$. In the calculation the $R=1$ subband, however, starts to be occupied only at $N_{\mathrm{s}} \approx 3.2 \times 10^{11} \mathrm{~cm}^{-2}$. By lowering $N_{\mathrm{A}}$ to $3 \times 10^{15} \mathrm{~cm}^{-3}$, one can lower the threshold for second subband occupation to around the experimental value. This suggests that some alteration of $N_{\mathrm{A}}$ from the nominal value may take place both during and after growth due to interdiffusion. In addition, the scatter of the experimental points around the theoretical curves indicates that $N_{\mathrm{A}}$ may differ from sample to sample. In spite of this the $\mathrm{Sb}_{4}$ flux method described in section 2 [12] has yielded lower values of $N_{\mathrm{s}}$ and fewer occupied subbands than previous attempts [14], and so is certainly worthwhile.

\subsection{Parallel-field magnetoresistance}

When a magnetic field is applied in the plane of a 2DEG, hybrid electric-magnetic subbands are formed, which shift upwards in energy with increasing magnetic field $[9,23]$. The parallel field acts most strongly on electric subbands with a large spatial extent, so that, in an inversion layer, the upper subbands will be pushed up in energy relative to the lower ones, resulting in their progressive depopulation until only the lowest is 


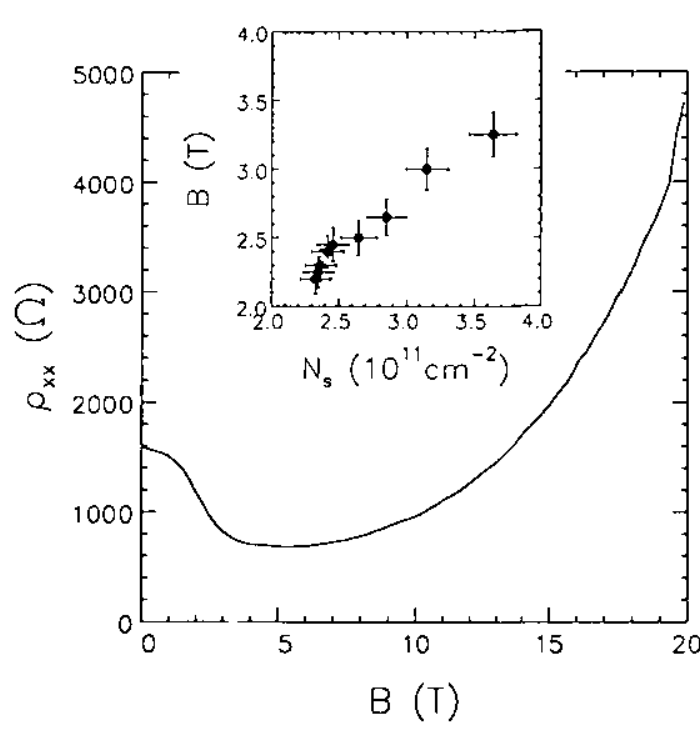

Figure 4. The parallel-field magnetoresistance of an InSb-CdTe heterojunction with $N_{s}=2.1 \times 10^{11} \mathrm{~cm}^{-2}$. The inset shows the depopulation field for the $R=1$ subband against $N_{s}$.

occupied. This depopulation can be seen directly in the resistance of the system, through its influence on the scattering -mechanisms of the subbands: once the bottom of the subband rises above the Fermi energy and is depopulated, the elastic and quasi-elastic processes that cause intersubband scattering can no longer take place, and the mobility of the system will increase, leading to a decrease in resistance $[9,23]$. The parallel-field magnetoresistance is thus useful for counting the number of occupied subbands and for examining the influence that each has on the scattering time of the carriers.

Typical parallel-field magnetoresistance data for one of the samples are shown in figure 4: a large negative magnetoresistance followed by a strong positive magnetoresistance is observed. The region of negative magnetoresistance is characteristic of the depopulation of a higher subband, as described above. If the field at which the negative magnetoresistance has its maximum slope is taken to mark the depopulation field [9], and this is measured as a function of $N_{\mathrm{s}}$ (varied using $\mathrm{PPC}$ ), the results lie on an approximately straight line as a function of $N_{\mathrm{s}}$ : this is shown as an inset to figure 4. This type of behaviour is again characteristic of the depopulation of a subband, and is due to the self-consistency of the potential $[23,24]$. Interestingly, the 'depopulation feature' is present even when there is a negligible second subband occupancy. Fletcher et al [25] have observed the onset of a reduction of mobility in GaAs-(Ga,Al)As heterojunctions at $2 \mathrm{D}$ carrier densities significantly below those at which the second subband was populated: this was attributed to the second subband being broadened by disorder, and possessing a 'tail' of localized states (or a mobility edge) which contributes to the scattering but which is unable to take part in the carrier transport. In a similar manner, the fact that the depopulation feature' in the InSb-CdTe heterojunctions extrapolates to zero magnetic field at an $N_{\mathrm{s}}$ far below that at which no second subband carriers can be detected (compare figure 3 and the inset to figure 4) indicates that the upper subband in these samples is sufficiently broadened to allow the band tail to strongly affect the scattering in the ground subband.

The size of the decrease in resistivity due to the depopulation is $\approx 50 \%$ of the total resistivity and indicates the importance of intersubband scattering; this is of importance in the determination of the zero-field spin splitting in the following section. If the scattering times restricting the mobility due to intersubband scattering and due to intrasubband scattering are assumed to sum according to Matthiessen's rule, the two can be seen to be roughly equal. The intersubband scattering is almost certainly mediated by ionized impurities at the interface [26]. Magnetotransport measurements carried out on InSb-CdTe heterojunctions subjected to high hydrostatic pressures [27] indicate that the states responsible for the presence of the 2DEG have a lower symmetry than bulk shallow donors and are probably interface states occupying a band of energy that starts $\sim 50$ $100 \mathrm{meV}$ above the InSb band edge at the InSb-CdTe interface; it is these ionized states that will mediate the intersubband scattering and give rise to transport and single-particle scattering times that are similar in size [25] (see next section).

As mentioned above, the depopulation structure is superimposed on a positive magnetoresistance, which is due to the in-plane field increasing the density of states available for intrasubband scattering [23]: this effect is expected to be stronger in narrow-gap systems than in wide-gap systems such as $\mathrm{GaAs}-(\mathrm{Ga}, \mathrm{Al}) \mathrm{As}$ because of the smaller effective mass of the former, as is indeed observed experimentally $[9,28]$.

Finally it should be noted that only one region of negative magnetoresistance is observed in parallel field for the InSb-CdTe samples, even at the highest values of $N_{\mathrm{s}}$. The parallel-field magnetoresistance is perhaps the most sensitive probe of subband occupation [9], and so it can be stated that only two subbands are occupied for all $N_{\mathrm{s}}$ in these InSb-CdTe heterojunctions.

\section{Spin splitting of the subbands at zero magnetic field}

\subsection{Experimental determination using quantum interference}

A prominent feature of the theoretical treatments of 2D systems in narrow-gap semiconductors has been the zero-field spin splitting of the subbands, which results from three distinct mechanisms-the $k^{3}$ term in the bulk dispersion relationship, the spin-orbit interaction in an asymmetric potential well, and the splitting due to the presence of the boundary between the different materials $[1-4,15,16]$. In narrow-gap systems with a strong electric field at the 2DEG, the second effect will be strongest [1]. There have been attempts to observe the effect in spin-resonance experiments on $\mathrm{GaAs}-(\mathrm{Ga}, \mathrm{Al}) \mathrm{As}$ heterojunctions [29] and InSb MIS 

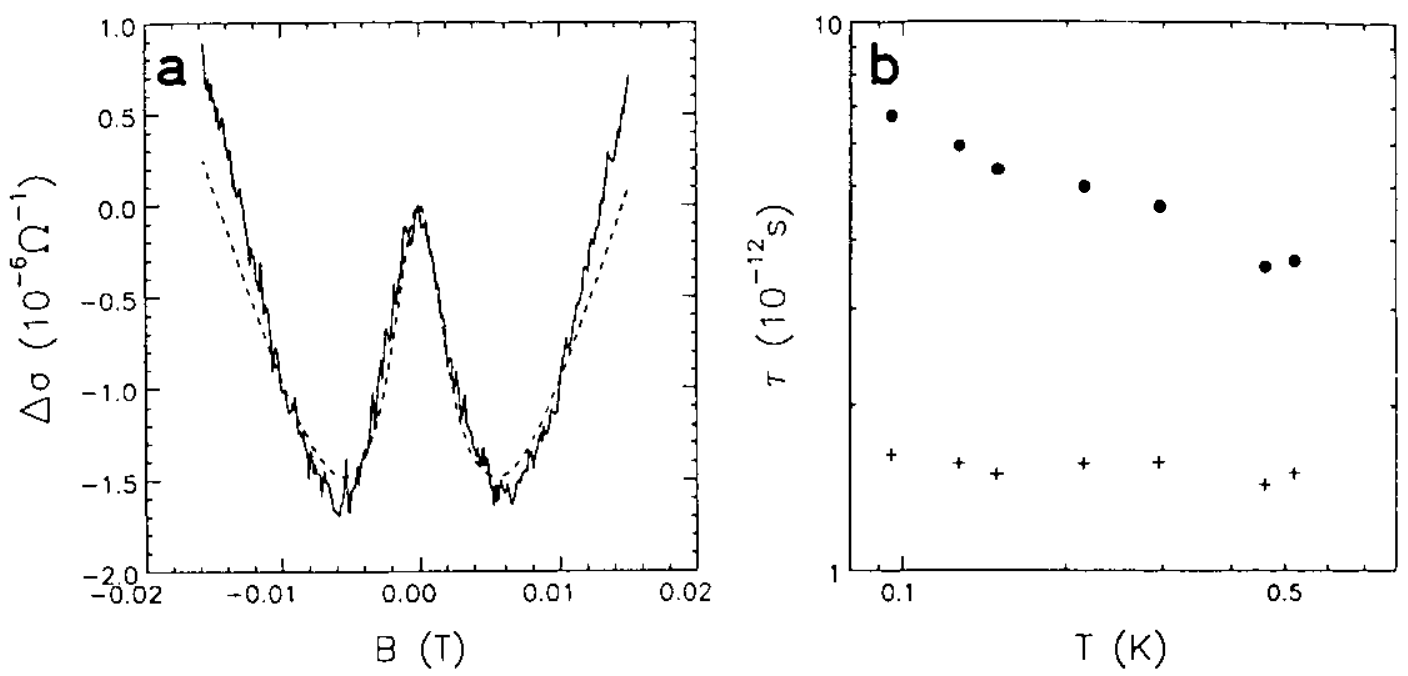

Flgure 5. (a) Low-field magnetoconductivity data for InSb-CdTe sample 1 (full curve) and a fit using equation (1) (broken curve). (b) The phase-breaking time $\tau_{\phi}(0)$ and spin-orbit scattering time $\tau_{s o}$ (t) against temperature for sample 1 .

structures [30]. However, the small size of the effect and the theoretical difficulty of extrapolating the measurements to zero field in the former case, and the rather weak electric field in the latter case, led to inconclusive results. More recently, a specially designed $\mathrm{p}-(\mathrm{Hg}, \mathrm{Cd}) \mathrm{Te}$ MIS structure combining large electric fields $\left(N_{\mathrm{A}}=10^{18} \mathrm{~cm}^{-3}\right.$, i.e. $\left.F \sim 2.5 \times 10^{4} \mathrm{~V} \mathrm{~cm}^{-1}\right)$ with a small band gap $\left(E_{\mathrm{g}}=150 \mathrm{meV}\right)$ was used to demonstrate that the effect can in fact be rather large [31]. A phenomenological approach was used to analyse the results, indicating that the spin splitting of the one occupied subband at the Fermi energy $E_{\mathrm{F}}$ in zero magnetic field could be around $13 \mathrm{meV}$. For convenience we shall refer to this zero-field spin splitting at $E_{\mathrm{F}}$ as $\Delta$. The electric field at the 2DEG in the InSb-CoTe samples can be estimated from the values of $N_{\mathrm{A}}$ obtained in section $2[5,26,31]$ to be $\sim 2 \times 10^{4} \mathrm{~V} \mathrm{~cm}^{-1}$, so that a reasonably large $\Delta$ is expected to be present.

In this work we use the low-field magnetoresistance to obtain the size of $\Delta$, a method pioneered by Gusev et al [32] in 2D hole gases. In this regime the magnetoresistance is dominated by the quantum interference corrections, which are strongly affected by the spin splitting. Figure 5(a) shows typical low-field diagonal magnetoconductivity $\left(\sigma_{x x}\right)$ data for an InSb-CdTe sample; the magnetoresistance is positive at the lowest fields and subsequently negative. This behaviour is characteristic of quantum interference in the presence of spin-orbit scattering [33].

Quantum interference is due to electrons scattering sequentially from a series of impurities so that they return to their starting point. Electrons can go around this scattering series in both directions, and, because the path is the same, electrons arriving back at their starting point after traversing the loop in both directions will be in phase with each other. The paths therefore interfere constructively at the starting point, increasing the probability of the electron returning to its start- ing point compared with the diffusive approximation, which increases the resistance above that in the Boltzmann approximation. Applying a magnetic field causes a phase difference between the two paths, removes the interference and therefore causes a negative magnetoresistance [34]. D'yakonov and Perel' [35] suggested a mechanism for spin-flip scattering, in which a finite spin splitting acts as a random magnetic field which rotates the electron's spin as it moves between impurities. This mechanism leads to antilocalization [36], because a spin rotated in real space by $2 \pi$ ends up in antiphasc with its starting state [37], so that the interference ends up on average destructive. The magnetoresistance is characterized by a spin-orbit scattering time $\tau_{\mathrm{so}}$ and a phase-breaking time $\tau_{\phi}$; the former is the expected time for a spin flip whereas the latter represents the time that an electron travels before it loses its phase. In samples where the spin-orbit scattering time $\tau_{\mathrm{so}}$ lies between $\tau_{t}$ (the transport scattering time from the mobility $\left.\mu=e \tau_{t} / m^{*}\right)$ and $\tau_{\phi}$, small loops, corresponding to short traversal times, have no spin-orbit scattering, and so are localized, whereas larger loops arc antilocalized. As the field increases, phase-breaking occurs in loops enclosing one flux quantum, so that the quantum interference in large loops is removed first, followed by that due to small loops: thus the magnetoresistance is first positive and then negative.

In order to estimate the size of $\Delta$ from data such as those shown in figure $5(a)$, we must first extract the two relevant scattering times, $\tau_{\phi}$ and $\tau_{\text {so }}$, which can be done using the theory of [33]. Later authors $[34,36]$ amended the result slightly to give the formula

$$
\begin{aligned}
& \Delta \sigma(B)=-\frac{e^{2}}{2 \pi^{2} \hbar}\left[\Psi\left(\frac{1}{2}+\frac{B+B_{\mathrm{so}}}{B}\right)\right. \\
& \left.+\frac{1}{2} \Psi\left(\frac{1}{2}+\frac{B_{\phi}}{B}\right)-\frac{3}{2} \Psi\left(\frac{1}{2}+\frac{\frac{4}{3} B_{\mathrm{so}}+B_{\phi}}{B}\right)\right]
\end{aligned}
$$

where $B$ is the magnetic field, $B_{\mathrm{so}}=\hbar / 4 e D \tau_{\mathrm{so}}$ and 
$B_{\phi}=\hbar / 4 e D \tau_{\phi} ; D$ is the diffusion constant and $\Psi$ is the digamma function.

A typical fit is shown in figure $5(a)$ as the broken curve: in general, the fits are good over a large field range at higher temperatures or with lower mobility samples, whereas for the highest mobility samples at the lowest temperatures the fit ceases to be good except at very low fields. Three features of the graph must be fitted (the $B^{2}$ coefficient at low fields, the coefficient of $\log (B)$ at higher fields and the field at which the turnover occurs) but there are only two parameters to vary $\left(\tau_{\phi}, \tau_{\text {so }}\right)$. Therefore this poor fit means that the theory is starting to fail at these fields and temperatures. The theory requires that the magnetic field be very small, given by $\omega_{\mathrm{c}} \tau_{\mathrm{t}} \ll 1 / E_{\mathrm{F}} \tau_{\mathrm{t}} \ll 1$ [38]. The first inequality becomes $\omega_{\mathrm{c}} \tau_{\mathrm{t}} E_{\mathrm{F}} \tau_{\mathrm{t}} \sim 0.3$ [42] for good samples at the magnetic fields used, which may well be too close to 1 for the formula to remain accurate.

The effects of a second subband are unimportant because the theory only requires that the samples are quasi-two-dimensional. Rainer and Bergmann [39] showed this explicitly for the case of multiple subbands and also explained how to interpret the scattering times obtained. In our samples there is strong intersubband scattering; this is shown by the large percentage fall in resistance when a subband depopulates due to a parallel magnetic ficld (figure 4). In such cases the times obtained are averages of the times in each subband, weighted according to the carrier density in each. Due to the low occupancy of the second subband, these times are almost the same as those for the lowest subband alone; i.e. our measurement will lead to a determination of $\Delta$ only for the lowest $(R=0)$ subband.

Figure $5(b)$ shows typical values of the phasebreaking scattering time $\tau_{\phi}$ and the spin-orbit scattering time $\tau_{\text {so }}$ obtained using the fits to the low-field magnetoconductance, plotted as a function of temperature. The figure shows that $\tau_{\text {so }}$ has no significant temperature dependence, which is expected because the spin-orbit scattering mechanisms do not depend on the temperature [39]. $\tau_{\phi}$ does depend on the temperature but remains greater than $\tau_{\text {so }}$; this sample therefore always fulfils the necessary condition for the observation of the W-shaped magnetoconductance.

Having found the scattering times, the spin splitting $\Delta$ can be related to the measured $\tau_{\text {so }}$ using the formula [35]

$$
\tau_{\mathrm{so}}^{-1}=\frac{2}{3} a \frac{\Delta^{2}}{\hbar^{2}} \tau_{\mathrm{t}}
$$

where the prefactor $a$ varies between 1 and $\frac{1}{6}$, and $\tau_{\mathrm{t}}$ is the transport scattering time derived from the mobility $\mu=e \tau_{t} / m^{*}$. When a significant fraction of the scattering is at large angles, $a$ is close to 1 . In order to estimate $a$, we have measured the ratio of $\tau_{\mathrm{s}}$, the single-particle scattering time derived from the fielddependent amplitude of the $\mathrm{SdH}$ oscillations [40], to $\tau_{\mathrm{t}}$ for the samples used in these measurements: e.g. for sample $1\left(N_{\mathrm{s}}=1.7 \times 10^{11} \mathrm{~cm}^{-2}\right) \tau_{\mathrm{s}} / \tau_{\mathrm{t}}=0.7 \pm 0.1$, while for sample $3\left(N_{\mathrm{s}}=2.0 \times 10^{11} \mathrm{~cm}^{-2}\right), \tau_{\mathrm{s}} / \tau_{\mathrm{t}}=0.3 \pm 0.1$. If the scattering is assumed to be screened Coulombic scattering, the measured ratios correspond to $a=0.6 \pm$ 0.1 , using the formula of D'yakonov and Perel'. This is characteristic of a slightly greater probability for smallangle scattering than for large-angle scattering.

A series of magnetoconductance runs were performed at $1.2 \mathrm{~K}$ using the PPC effect to vary the carrier density and hence the Fermi wavevector. The spin splitting $\Delta$ was calculated using equation (2) from the scattering times obtained using equation (1); the values are plotted in figure 6 as a function of $N_{\mathrm{s}}$. The error bars are mainly due to the uncertainty in the value of the parameter $a$ in equation (2). Within the limits of the experimental errors, $\Delta$ has values between 2 and $4 \mathrm{meV}$, and increases with increasing $N_{\mathrm{s}}$. Having seen that the zero-field spin splitting is a significant effect, we shall now calculate the size of the contributions to $\Delta$ mentioned above and compare them with the experimental results.

\subsection{Theoretical estimate of the spin splitting}

As mentioned above, there are three main sources of zero-field spin splitting in narrow-gap semiconductorsthe $k^{3}$ term arising from the bulk anisotropy, the linear$k$ term due to the asymmetry of the electric field in the 2D system and the splitting due to the presence of the boundary between the two different materials (also linear in $k$ ). The three-band model takes the two latter contributions into account directly (not by a perturbation technique) $[15,16]$. On the other hand, the $k^{3}$ term, which clearly lies outside the scope of the model, is not included, even as a perturbation. The reason behind this decision follows Rössler's argument that the $k^{3}$ term is of lesser importance in narrow:gap semiconductors than in e.g. GaAs [1]. The calculated results for the $R=0$ and $R=1$ subbands are shown in figure 6 as curves; the agreement with the data (points), which give the splitting of the dominant $R=0$ subband, is quite reasonable, improving for higher values of $N_{\mathrm{A}}$. However, the curves calculated using the lower values of $N_{\mathrm{A}}$ which give the best agreement between the predicted and measured subband occupancies (see section 4.1 and figure 3 ) lie below the experimental values for $\Delta$; in addition the calculations show a less steep variation with $N_{\mathrm{s}}$ than the data do.

In order to assess whether the inclusion of the splitting due to the $k^{3}$ term would improve the agreement, we can make a separate estimate of its size; it is the term that breaks the Kramer's degeneracy between spinup and spin-down states in the Hamiltonian of a bulk semiconductor, and is given by [41]

$\Delta_{k^{3}}= \pm C_{1} k^{2}\left[k^{2}\left(k_{x}^{2} k_{y}^{2}+k_{y}^{2} k_{z}^{2}+k_{z}^{2} k_{x}^{2}\right)-9 k_{x}^{2} k_{y}^{2} k_{z}^{2}\right]$.

Here $C_{1}=4 E_{\Delta} P \beta / 3 E_{\mathrm{g}}\left(E_{\mathrm{g}}+E_{\Delta}\right), E_{\Delta}$ is the separation of the spin-orbit split-off band and the valence band extrema, $P$ is a band structure parameter tabulated in [42] and $\beta$ is a constant that has been calculated from band structure parameters [43] and measured experimentally [44]; both methods obtained $|\beta|=3.3 \hbar^{2} / 2 m^{*}$. The variation of $\Delta_{k^{3}}$ with 


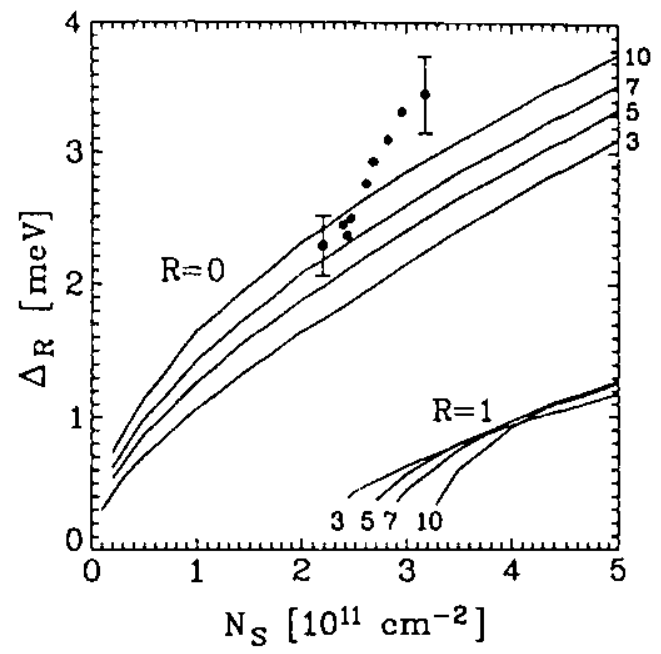

Figure 6. Experimental values of $\Delta$ against $N_{s}$ (points) compared with theory. Results of the self-consistent calculations of $\Delta$ (only linear-k terms evaluated) are shown as curves for several different values of $N_{A}$ (units as in figure 3) and for both the $R=0$ and $R=1$ subbands.

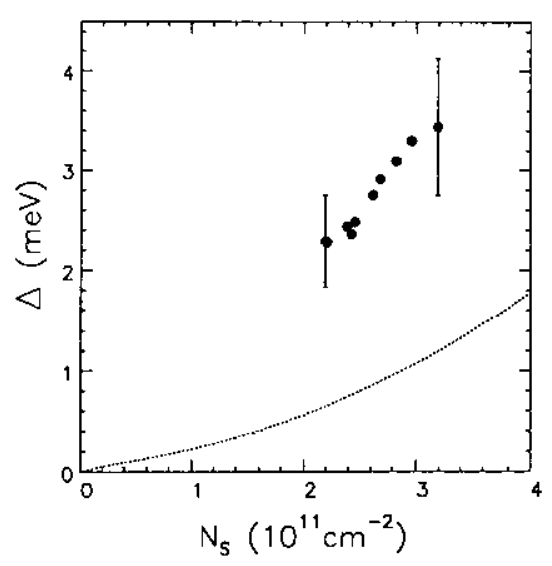

Figure 7. Experimental values of $\Delta$ against $N_{8}$ (points) compared with calculations of $\Delta_{k^{3}}$ evaluated using equation (3) (dotted curve).

$N_{\mathrm{s}}$ predicted by equation (3) is shown alongside the data in figure 7. The calculated $k^{3}$ term can be seen to have roughly the correct size and $N_{\mathrm{s}}$ dependence to qualitatively improve the agreement between the data and the self-consistent calculations shown in figure 6. However, one should be cautious in e.g. simply adding the two terms; the three contributions mix the states in different ways and the resulting total splitting is not a simple sum.

\section{Summary}

We have studied a series of InSb-CdTe heterojunctions containing a high-mobility 2 DEG with a low carrier density and only one or two occupied subbands. These de- sirable qualities have been obtained by using improved molecular beam epitaxy techniques and by growing the structure on an InSb substrate with $N_{\mathrm{A}}=10^{16} \mathrm{~cm}^{-3}$ to ensure a large electric field at the interface. In addition, the convenient similarity of the crystal and band structures of the two materials has allowed self-consistent calculations of the subband energy structure to be performed.

The 2DEG exhibits well resolved quantum Hall plateaux and Shubnikov-de Haas oscillations at high magnetic fields and temperatures below $5 \mathrm{~K}$; using these data and the persistent photoconductivity (PPC) effect, we have evaluated the subband occupancies as a function of $2 \mathrm{D}$ carrier density $N_{\mathrm{s}}$. In addition, parallel-field magnetoresistance measurements show that only two subbands are occupied, even at the highest values of $N_{\mathrm{s}}$, and indicate the importance of intersubband scattering, thought to be mediated by interface defects. The self-consistent calculations predict the number of occupied subbands correctly, and are in good agreement with the experimental subband occupancies when a value of $N_{\mathrm{A}}$ around a factor of three lower than the nominal substrate concentration is used. This probably indicates that some interdiffusion between the InSb and CdTe has taken place, resulting in partial type-conversion of the InSb. Evidence for interdiffusion even at room temperature is also provided by the 2D carrier density of the samples, which increases with storage in the laboratory.

The low-field magnetoresistance of the heterojunctions at low temperatures is at first positive and then negative; this is characteristic of weak localization in the presence of spin-orbit scattering. An analysis of the data indicates that this is caused by a spin splitting of the subbands at the Fermi energy, present even in zero magnetic field, and of order $2-4 \mathrm{meV}$. The splitting results from three distinct mechanisms, two of which, proportional to $k$, are included exactly in the self-consistent calculations. The calculations reproduce the size of the observed splitting reasonably well, indicating that these linear- $k$ terms are the dominant contributions to the effect. The term proportional to $k^{3}$, not included in the self-consistent model, has also been estimated, and has a size $(\sim 1 \mathrm{meV})$ and $N_{\mathrm{s}}$ dependence which would improve the agreement between the self-consistent calculations and the data, although a simple addition of the two terms is not possible. These results illustrate the importance of the zero-field spin splitting in narrow-gap space-charge layers.

These preliminary measurements have demonstrated that the InSb-CdTe heterojunction is both an interesting system in its own right and an ideal tool with which to study effects specific to narrow-gap semiconductor space-charge layers. It is hoped that the improved growth techniques responsible for the samples in this study will lead to an upsurge of interest in this system.

\section{Acknowledgments}

This work was funded by the SCIENCE programme of 
the European Community, with additional support from The Netherlands Foundation for Fundamental Research on Matter (FOM), de Nederlandse Organisatie voor Wetenschappelijk Onderzoek (NWO) and the United Kingdom Science and Engineering Research Council (SERC).

\section{References}

[1] Lommer G, Malcher F and Rössler U 1988 Phys. Rev. Lett. 60728

[2] Takada Y, Arai K and Uemura Y 1982 Springer Lecture Notes àt Physics wol 152 (Berlin: Springer) p 101

[3] Zawadzki W 1983 J. Phys. C.: Solid State Phys. 16229

[4] Ando T 1985 J. Phys. Soc. Japan 542676

[5] Merkt U, Horst M, Evelbauer T and Kotthaus J P 1986 Phys. Rev. B 347234

[6] Koch F 1986 Optical Properties of Narrow-Gap LowDimensional Structures ed C M Sotomayor-Torres et al (NATO ASI Series 152) (New York: Plenum) p 187

[7] Gobsch G, Paasch G, Schulze D, Handschack S and Fiedler T 1988 Solid State Commun. 651583

[8] Grabecki G, Suski T, Dietl T, Skoskiewicz T and Glinski M 1987 Proc. Conf. on High Magnetic Fields in Semiconductor Physics (Wirzburg, 1986) ed G Landwehr (Springer Series in Solid State Sciences 71) (Berlin: Springer) p 127

[9] Nasir F, Singleton J and Nicholas R J 1988 Semicond. Sci. Technol. 3655

[10] van Welzenis R G and Ridley B K 1984 Solid-State Electron. 27113

[11] Williams G M, Whitehouse C R, Chew N G, Blackmore G W and Cullis A G $1985 \mathrm{~J}$. Vac. Sci. Technol. B 3704

[12] Golding T D, Greene S K, Pepper M, Dinan J H, Cullis A G, Williams $G M$, Barnett $S \mathrm{~J}$ and Whitehouse C R 1990 Semicond. Sci. Technol. 5 S311

[13] Zheng Y D, Chang Y H, McCombe B D, Farrow R F C, Temofonte T and Shirland F A $1986 \mathrm{Appl}$ Phys. Lett. 491187

[14] Grimes R T, Janssen T J B M, Stanaway M B, Langerak C J G M, Singleton J, Ashenford D E, Lunn B, Hill G and Chamberlain J M 1992 Proc. 9th Int. Conf. on Electronic Properties of Two Dimensional Systems (Nara, 1991) (Surf. Sci. in press)

[15] Sobkowicz P 1990 J. Cryst. Growth 101 337; Semicond. Sci. Technol. 5183

[16] Nachev I 1988 Semicond. Sci. Technol. 3 29; Phys. Scr. 37825

[17] Greene S K 1990 PhD Thesis University of Cambridge

[18] Kirk W P, Kobiela P S, Shih H D and Reed M A 1987 Proc. Conf. on High Magnetic Fields in Semiconductor Physics (Würzburg, 1986) ed G Landwehr (Springer Series in Solid State Sciences 71) (Berlin: Springer) p 122
[19] Prange R E and Girvin S E (ed) 1987 The Quantum Hall Effect (New York: Springer)

[20] Nicholas R J, Brummell M A and Portal J-C 1984 J. Cryst. Growth 68356

[21] Kane M J, Apsley N, Anderson D A, Taylor L L and Kerr T 1985 J. Phys. C: Solid State Phys. 185629

[22] Singleton J, Nasir F and Nicholas R J 1986 Proc. SPIE 65999

[23] Tang Hui and Butcher P N 1988 J. Phys. C: Solid State Phys. 213313

[24] Reisinger H and Koch F 1986 Surf. Sci. 170397

[25] Fletcher R, Zaremba E, D'Iorio $M$, Foxon C T and Harris J J 1988 Phys. Rev. B 387866

[26] Ando T, Fowler A B and Stern F 1982 Rev. Mod. Phys. 54437

[27] Singleton J, Greene S K, Golding T D, Pepper M, Skierbiszewski C, Wisniewski P, van der Wel $P$ J, van Thor P H E and Dinan J 1991 Superlatt. Microstruct. 951

[28] Harris J J, Lacklison D E, Foxon C T, Selten F M, Suckling A M, Nicholas R J and Barnham K W J 1987 Semicond. Sci. Technol. 2783

[29] Stein D, von Klitzing K and Weimann G 1983 Phys. Rev. Lett. 51130

Dobers M, won Klitzing $\mathrm{K}$ and Weimann G 1988 Phys. Rev. B 385453

[30] Därr A, Kotthaus J P and Ando T 1977 Proc. 13th Int. Conf. on the Physics of Semiconductors (Rome, 1976) ed F G Fumi (Amsterdam; North-Holland) p 774

[31] Wollrab R, Sizmann R, Koch F, Ziegler J and Maier H 1989 Semicond. Sci. Technol. 4491

[32] Gusev G M, Kvon Z D and Ovsyuk V N 1984 J. Phys. C: Solid State Phys. 17 L683

[33] Hikami S, Larkin A I and Nagaoka Y 1980 Prog. Theor. Phys. 63707

[34] Bergmann G 1983 Phys. Rep. 1071

[35] D'yakonov M I and Perel' V U 1971 Zh. Eksp. Teor. Fiz. 861784 (Sov. Phys. JETP 59 1053); 1972 Fiz. Tverd. Tela. 133581 (Sov. Phys. Solid State 13 3023)

[36] Al'tshuler B L and Aronov A G $1981 \mathrm{Zh}$. Eksp. Teor. Phys. 81763 (Sov. Phys. JETP 54 411)

[37] Bergmann G 1982 Solid State Commun. 42815

[38] Kawabata A 1984 J. Phys. Soc. Japan 533540

[39] Rainer D and Bergmann G 1985 Phys. Rev. B 323522

[40] Coleridge P T, Stoner R and Fletcher R 1989 Phys. Rev. B 391120

[41] Kittel C 1963 Quantum Theory of Solids (New York: Wiley)

[42] Kane E O 1979 Proc. Conf. Int. Summer School, Nimes ed W Zawadzki (Springer Lecture Notes in Physics 133) (Berlin: Springer)

[43] Alferove Zh I, Gorelenlok A T, Mamutin V V, Polyanskya T, Savel'ev I G and Shmartsev Yu V 1984 Fiz. Tech. Poluprovodn. 181999 (Sov. Phys. Semicond. 18 1247)

[44] Seiler D G, Bajaj B D and Stephens A E 1977 Phys. Rev. B 162822 